\title{
Optimal Worst-Case QoS Routing in Constrained AWGN Channel Network
}

\author{
Edwin Soedarmadji Robert J. McEliece \\ California Institute of Technology, Pasadena, CA 91125, USA, \\ edwin@systems.caltech.edu
}

\begin{abstract}
In this paper, we extend the optimal worst-case QoS routing algorithm and metric definition given in [1]. We prove that in addition to the $q$-ary symmetric and $q$-ary erasure channel model, the necessary and sufficient conditions defined in [2] for the Generalized Dijkstra's Algorithm (GDA) can be used with a constrained non-negative-mean AWGN channel.

The generalization allowed the computation of the worst-case QoS metric value for a given edge weight density. The worst-case value can then be used as the routing metric in networks where some nodes have error correcting capabilities.

The result is an optimal worst-case QoS routing algorithm that uses the Generalized Dijkstra's Algorithm as a subroutine with a polynomial time complexity of $O\left(V^{3}\right)$.
\end{abstract}

\section{INTRODUCTION}

Mission critical communication networks have very high requirements for performance and reliability. These networks are designed to minimize the possibility of losing even one symbol, packet, or file. Optimizing the Quality of Service (QoS) of such networks requires evaluating network paths (and edges) and selecting those paths and edges optimality that minimizes the worst possible number of observed undesired event (which could be loss, error, etc.). In many applications, such as ad-hoc wireless (sensor) networks, this type of QoS optimization is both critical, and yet highly untrivial.

A novel QoS metric called the worst-case error (or erasure) metric (WCE) was introduced in [1], where it was used to measure, compare, and select the network path whose WCE length is minimum. The WCE metric is derived from the metric more commonly used in specifying communication channel qualities: the Bit Error (or Erasure) Ratio (BER).

In the original introduction, only the cases of $q$-ary Symmetric Channel and $q$-ary Erasure Channel were analyzed. It was shown that for these channels, the edge WCE length is a non-decreasing function of its BER length. Thus the optimal BER path is also the optimal WCE path, and vice versa.

In this paper, we extend the optimal worst-case QoS analysis to cover a special case of AWGN channel that has non-negative mean and a constraint on its mean to variance ratio. This channel can be easily used to model delay channel, or in some cases, the Gaussian approximation of Poisson-distributed channels. To calculate a path's BER length from the edges' BER lengths, we use the same algebra as in [1]. The path BER was then applied to the Generalized Dijkstra's Algorithm.

This work was supported by the Caltech Lee Center for Advanced Networking and NSF Grant No. CCF-0514881
We show that if some network nodes are capable of correcting erasures, it is often possible to find a path (or paths) with zero WCE length. This partial error correction capability is reasonable. In many wireless and ad-hoc networks, energy (and ultimately compute power) is limited.

Consequently, available resource for error correction that involves complex mathematical operations such as finite fields arithmetic and iterative algorithms is limited as well. In addition, error correction incurs delay and consumes bandwidth. Hence, it is desirable to limit the number of error-correcting nodes. Our algorithm optimizes the worst-case QoS by routing information through the error-correcting nodes.

Forward Error Correction (FEC) is also gaining acceptance in modern networks. Historically, error correction over wired networks has predominantly used the Automatic Repeat reQuest (ARQ) methods over the FEC methods. However, in wireless multimedia applications, FEC outshines ARQ in its ability to significantly improve network QoS [3] [4] [5] [6] without a heavy premium on performance. Unlike TCP and ARQ, FEC does not use return requests and thus consumes less bandwidth [7], especially in large multicast networks typical of wireless multimedia applications [8] [9].

Even in peer-to-peer networks, FEC deployed at strategically positioned error-correcting nodes is superior compared to replication [10]. Multicast algorithms such as Digital Fountain [11] and Bullet [12] employ FEC-based codes. Adaptive [5] [6] and hybrid (ARQ-FEC) QoS-driven algorithms that dynamically adjust FEC level to network conditions have been proposed [13] [14] [15] [16] to reduce the bandwidth and computation overhead of FEC methods.

Our analysis proves that the worst-case QoS routing metric and algorithm can be applied to a wide class of continuous AWGN channels, which are very versatile and flexible. Many other (QoS) network optimization problems can be transformed into this problem. The algorithm is particularly useful for minimizing the worst-case occurence probability of the "extreme" events that are non-typical, but nevertheless highly catastrophic.

In the next section, we provide a problem formulation. Our notation generalized the previous results which are specific to $q$-ary channels. In the same section, we define the constrained AWGN channel. In the third section, we prove that the constrained AWGN channel is compatible with the GDA. Finally, we describe the optimal worst-case QoS routing algorithm before concluding with discussions. 


\section{Formulation AND NOTATION}

We model the network as a digraph $G=(V, E)$, where the node, edge and path sets of $G$ are denoted by $V, E$, and $\Pi$. The nodes $s$ and $d \in V$ are the source and destination nodes, and $\Pi \subset \Pi$ is the set of all paths from $s$ to $d$.

A path $\pi \in \Pi$ whose nodes $V_{\pi} \subset V$ are connected by $E_{\pi} \subset E$ is denoted by either $\left\langle v_{0}, \ldots, v_{J}\right\rangle,\left\langle e_{1}, \ldots, e_{J}\right\rangle$, or $\left\langle v_{0}, e_{1}, \ldots, e_{J}, v_{J}\right\rangle$. The number of nodes (or edges) in $\pi$ is denoted by $|\pi|_{v}$ (or $|\pi|_{e}$ ). The symbol $\left\langle v_{i}, v_{i+1}\right\rangle$ denotes the edge (path) connecting the two (non-) adjacent nodes $v_{i}$ and $v_{i+1}$. A partial path $\pi_{j}$ of $\pi$ denotes $\left\langle v_{0}, \ldots, v_{j}\right\rangle$, with $0<$ $j \leq J$, and a truncated path $\bar{\pi}_{j}$ is $\left\langle v_{0}, e_{1}, \ldots, v_{j-1}, e_{j}\right\rangle$.

Denote the message by $B \in \mathcal{B}$, where $\mathcal{B}$ is the space of all allowable messages in the network. Let $B_{i}$ denote the value of $B$ as it departs from $v_{i}$; and let $\bar{B}_{i}$ denote the value of $B$ as it leaves $e_{i}$. Both $v_{i}$ and $e_{i}$ are parts of $\left\langle v_{0}, e_{1}, \ldots, e_{J}, v_{J}\right\rangle$, along which $B$ evolves as follows:

$$
B_{0} \stackrel{e_{1}}{\longrightarrow} \bar{B}_{1} \stackrel{v_{1}}{\longrightarrow} B_{1} \stackrel{e_{2}}{\longrightarrow} \bar{B}_{2} \stackrel{v_{2}}{\longrightarrow} \cdots \stackrel{e_{J}}{\longrightarrow} \bar{B}_{J} \stackrel{v_{J}}{\longrightarrow} B_{J} .
$$

Here $v_{i}$ and $e_{i}$ can be regarded as operators $\boldsymbol{v}_{i}, \boldsymbol{e}_{i} \in \mathcal{E}: \mathcal{B} \rightarrow \mathcal{B}$ given by $B_{i}=\boldsymbol{v}_{i}\left(\bar{B}_{i}\right)$ and $\bar{B}_{i+1}=\boldsymbol{e}_{i}\left(B_{i}\right)$. For $\pi$, the evolution operator is $\boldsymbol{\pi}=\boldsymbol{v}_{J} \circ \boldsymbol{e}_{J} \circ \cdots \circ \boldsymbol{e}_{1} \circ \boldsymbol{v}_{0}$, for $\pi_{j}$, it is $\boldsymbol{\pi}_{j}=$ $\boldsymbol{v}_{j} \circ \boldsymbol{e}_{j} \circ \cdots \circ \boldsymbol{e}_{1} \circ \boldsymbol{v}_{0}$, and for $\bar{\pi}_{j}$, it is $\overline{\boldsymbol{\pi}}_{j}=\boldsymbol{e}_{j} \circ \boldsymbol{v}_{j-1} \cdots \circ \boldsymbol{e}_{1} \circ \boldsymbol{v}_{0}$. Thus, $\boldsymbol{\pi}_{j}\left(B_{0}\right)=B_{j}$ and $\overline{\boldsymbol{\pi}}_{j}\left(B_{0}\right)=\bar{B}_{j}$.

Define $X: \mathcal{B} \times \mathcal{B} \rightarrow \mathcal{M}$ as a function measuring the Hamming distance $x \in \mathcal{M}$ between a message $B_{0}$ at $v_{0}$ which evolves into $B_{J}$ at $v_{J}$; where $v_{0}$ and $v_{J}$ are connected by $\left\langle v_{0}, v_{J}\right\rangle$, and $\mathcal{M}$ is the metric space. The distance $x$ from $B_{i}$ to $B_{i^{\prime}}$ is denoted by $x=X_{i, i^{\prime}}=X\left(B_{i}, B_{i^{\prime}}\right)$. Let $X_{i, \bar{i}^{\prime}}$ denote $X\left(B_{i}, \bar{B}_{i^{\prime}}\right)$. If $e=\left\langle v_{i}, v_{i^{\prime}}\right\rangle$ then we define the shorthand $X(e)=X\left(B_{i}, \bar{B}_{i^{\prime}}\right)$.

Besides $B_{0}$, the messages $B_{i}$ at $v_{i}$ are random variables. Therefore, $X_{0, i}$ are also random variables. Consider $x=$ $X_{i, i^{\prime}}$, the random variables measuring the distance between the message at $v_{i}$ and its image at $v_{i^{\prime}}$. Define $P(x, \boldsymbol{\lambda})$ as the probability density of $x$ parameterized by a vector $\boldsymbol{\lambda} \in \Lambda$. For example, for $x \in \mathbb{R}$ and $P$ Gaussian, $\lambda$ is the vector of $P$ 's mean and variance $\left(\mu, \sigma^{2}\right)$. The value $\boldsymbol{\lambda}=\infty$ denotes the absence of connection between two nodes. Each edge $e_{i}$ in the network can be characterized by its parameter $\boldsymbol{\lambda}_{i}$.

Suppose the nodes $U \subseteq V$ can reset $x\left(\pi_{j}\right)$ of any path $\pi_{j}$ that passes through $v_{j} \in U$, as long as $x\left(\pi_{j}\right)<x_{\max }$.

$$
\begin{aligned}
X_{0, j} & =0 & & , v_{j} \in U \text { and } X_{0, \bar{j}} \leq x_{\max } \\
& \geq X_{0, \bar{j}} & & , v_{j} \in U \text { and } X_{0, \bar{j}}>x_{\max } \\
& =X_{0, \bar{j}} & & , v_{j} \in V \backslash U
\end{aligned}
$$

We also assume that $d \in U$. For a path $\pi$, if $V_{\pi} \cap U \neq \varnothing$ then $X_{0, j}$ is a non-decreasing function of $j$.

Finally, we define the worst-case function $\bar{x}\left(\boldsymbol{\lambda}_{i}, \epsilon\right)$ that computes the worst-case "possible" value of $x$, where "possible" values $y$ are defined as those $y$ with $P\left(y, \boldsymbol{\lambda}_{i}\right)>\epsilon$.

$$
\bar{x}\left(\boldsymbol{\lambda}_{i}, \epsilon\right)=\max _{x}\left\{x \mid P\left(x, \boldsymbol{\lambda}_{i}\right) \geq \epsilon, x \in \mathcal{M}\right\}
$$

In general, $\bar{x}(\cdot)$ is a functional accepting $P(x)$. However, here we focus on probability densities with well-defined parameters.
Consider a path $\pi=\left\langle e_{1}, \ldots, e_{J}\right\rangle \in \Pi$ and its partial path $\pi_{j}=\left\langle e_{1}, \ldots, e_{j}\right\rangle$ with $1 \leq j \leq J$. For convenience, we also define the function $\beta: \Pi \rightarrow \Lambda$ that maps a path $\pi$ (or an edge $e_{i}$ ) into a density parameter $\boldsymbol{\lambda}_{\pi}$ (or $\boldsymbol{\lambda}_{i}$ ) and the function $\omega: \Pi \rightarrow \mathcal{M}$ that maps a path or an edge (given $\epsilon$ ) into its worst-case value $x \in \mathcal{M}$.

For $e_{i}$, the $\beta$ and $\omega$ are related to $\bar{x}\left(\boldsymbol{\lambda}_{i}, \epsilon\right)$ through: $\omega\left(e_{i}\right)=$ $\bar{x}\left(\beta\left(e_{i}\right), \epsilon\right)$. For $\pi$, assuming $\boldsymbol{\lambda}_{\pi}$ is defined, similarly we have $\omega(\pi)=\bar{x}(\beta(\pi), \epsilon)$. The next question is, how does $\boldsymbol{\lambda}_{\pi}$ depend on $\boldsymbol{\lambda}_{i}$ 's, and how does $\bar{x}\left(\boldsymbol{\lambda}_{\pi}, \epsilon\right)$ depend on $\bar{x}_{i}=\bar{x}\left(\boldsymbol{\lambda}_{i}, \epsilon\right)$ ?

The quantities $x, \boldsymbol{\lambda}$, or $\bar{x}$ all have the potential to be used as the routing metric. However, in general, $x_{\pi} \neq \sum x_{i}, \boldsymbol{\lambda}_{\pi} \neq$ $\sum \boldsymbol{\lambda}_{i}$, and $\bar{x}_{\pi} \neq \sum \bar{x}_{i}$, where $\sum$ is the standard scalar or vector summation. Let us assume that the addition operation is defined in $\Lambda$ and $\mathcal{M}$ and is denoted by $\oplus$. If $x_{1}=X\left(e_{1}\right), x_{2}=$ $X\left(e_{2}\right), \boldsymbol{\lambda}_{1}=\beta\left(e_{1}\right), \boldsymbol{\lambda}_{2}=\beta\left(e_{2}\right), \bar{x}_{1}=\omega\left(e_{1}\right), \bar{x}_{2}=\omega\left(e_{2}\right)$, and $\pi=\left\langle e_{1}, e_{2}\right\rangle$, then we say $x_{\pi}=x_{1} \oplus x_{2}, \boldsymbol{\lambda}_{\pi}=\boldsymbol{\lambda}_{1} \oplus \boldsymbol{\lambda}_{2}$, or $\bar{x}_{\pi}=\bar{x}_{1} \oplus \bar{x}_{2}$. To be useful in a shortest-path algorithm, $\oplus$, $\Lambda$ and $\mathcal{M}$ have to obey certain algebraic properties.

In the next section, we outline these algebraic properties and how these properties can be used to test whether a certain routing metric is "compatible" with the GDA, in a sense that the GDA will not produce a loop.

With $\oplus$, we can now define $x_{\pi}, \boldsymbol{\lambda}_{\pi}$ and $\bar{x}_{\pi}$ in terms of $x_{i}, \boldsymbol{\lambda}_{i}$ and $\bar{x}_{i}$ using a generalized summation: $x_{\pi}=\bigoplus x_{i}$, $\boldsymbol{\lambda}_{\pi}=\bigoplus \boldsymbol{\lambda}_{i}$ and $\bar{x}_{\pi}=\bigoplus \bar{x}_{i}$. The pairings of $\Lambda$ and $\mathcal{M}$ with $\oplus$ form algebraic structures which we call the $\mathbf{X}, \mathbf{B}$, and $\mathbf{W}$ algebras, from the $X, \beta$, and $\omega$ functions, respectively.

Between two nodes, the optimal path $\pi^{*}$ is the path with the "shortest" path length from $s$ to $d$ when measured in the $\mathbf{X}, \mathbf{B}$ or $\mathbf{W}$ algebra (or metric). However, having $\oplus, X, \beta$, and $\omega$ is not enough to calculate $\pi^{*}$. We need to compare path lengths. Therefore we need a total order $\preceq$ on $\Lambda$ and $\mathcal{M}$ to evaluate expressions like $x_{\pi} \preceq x_{\pi^{\prime}}, \boldsymbol{\lambda}_{\pi} \preceq \boldsymbol{\lambda}_{\pi^{\prime}}$, or $\bar{x}_{\pi} \preceq \bar{x}_{\pi^{\prime}}$. Once $\preceq$ is defined, then we can define these optimal values for $G$ :

$$
\begin{aligned}
x^{*} & =\min _{\pi}\left\{x_{\pi} \mid \pi \in \Pi\right\} \\
\boldsymbol{\lambda}^{*} & =\min _{\pi}\left\{\boldsymbol{\lambda}_{\pi} \mid \pi \in \Pi\right\} \\
\bar{x}^{*} & =\min _{\pi}\left\{\bar{x}_{\pi}=\bar{x}\left(\boldsymbol{\lambda}_{\pi}, \epsilon\right) \mid \pi \in \Pi\right\}
\end{aligned}
$$

Preferrably, we would like to have the minima $\boldsymbol{\lambda}^{*}$ and $\bar{x}^{*}$ related by the expression: $\bar{x}^{*}=\bar{x}\left(\boldsymbol{\lambda}^{*}, \epsilon\right)$. Indeed, this is true not only for the $q$-ary symmetric channel and $q$-ary erasure channel [1], but also for the constrained AWGN channel we present here.

Later in this paper, we prove that if $V_{\pi} \cap U=\varnothing$, then the lengths $x_{\pi_{j}}, \boldsymbol{\lambda}_{\pi_{j}}$ and $\bar{x}_{\pi_{j}}$ are non-decreasing functions of $j$. This means that on any path, the metric $X_{0, j}$ increases as $j$ increases. In this case, reliable communication (in the worstcase sense) becomes very difficult to achieve except when the values $\boldsymbol{\lambda}_{i}$ are simultaneously favorable (which could represent a very small delay or error probability).

Often, this stringent level of QoS could be prohibitively difficult to achieve, forcing the engineers to settle for an unacceptably high catastrophic event probability. However, with $U$, we later show that with our algorithm, even a zero $\epsilon$-worstcase path is well within reach. 


\section{Non-negative-mean AWGN}

In this example, the message is a scalar $B \in \mathbb{R}^{+}$with non-negative-mean AWGN on each link. This message could represent the amount of non-negative degradation a packet has experienced so far. Therefore, the source always transmits $B_{0}=0$. At each node $v_{i} \in V_{\pi}$, two decisions are made: if $x_{i}=B_{i}-B_{0}$ exceeds $x_{\max }$, then the message (or the packet corresponding to the message) is discarded, otherwise it is retransmitted. Let us also assume that the nodes $u_{i} \in U \subseteq V$ can reset $B$ (and $x$ ) back to 0 .

The AWGN is characterized by $\boldsymbol{\lambda}=\left(\mu \geq 0, \sigma^{2}\right)$, where $\mu$ is the mean, and $\sigma^{2}$ the variance of the Gaussian density and the transition probability density is given by:

$$
\begin{aligned}
P\left(B_{i+1} \mid B_{i}\right) & =P\left(x ; \mu, \sigma^{2}\right) \\
& =\frac{1}{\sqrt{2 \pi \sigma^{2}}} \exp \left(\frac{-(x-\mu)^{2}}{2 \sigma^{2}}\right)
\end{aligned}
$$

where $x=B_{i+1}-B_{i}$. The $\mathbf{B}$ algebra is defined as follows. Two independent, adjacent edges $e_{1}$ and $e_{2}$ with parameters $\lambda_{1}$ and $\lambda_{2}$ can be treated as a single edge with parameter $\boldsymbol{\lambda}=$ $\boldsymbol{\lambda}_{1} \oplus \boldsymbol{\lambda}_{2}=\boldsymbol{\lambda}_{1}+\boldsymbol{\lambda}_{2}$, where the + sign is the standard vector summation operator. Since $x$ is now continuous, we can solve for $\bar{x}$ analytically by solving

$$
\bar{x}=P^{-1}\left(\epsilon ; \mu, \sigma^{2}\right)=\mu+\sqrt{-\sigma^{2} \ln \left(2 \pi \epsilon^{2} \sigma^{2}\right)},
$$

which has one solution if $2 \pi \sigma^{2}=\epsilon^{-2}$, two solutions $\bar{x}^{-}$and $\bar{x}^{+}$(with $\bar{x}^{-}<\bar{x}^{+}$) if $2 \pi \sigma^{2}<\epsilon^{-2}$, and no solution if $2 \pi \sigma^{2}>$ $\epsilon^{-2}$. Since $\bar{x}$ represents the worst-case value for $x$, we always assume $\bar{x}=\bar{x}^{+}$. From this result, we can also compute:

$$
\bar{x}_{1} \oplus \bar{x}_{2}=P^{-1}\left(\epsilon ; \mu_{1}+\mu_{2}, \sigma_{1}^{2}+\sigma_{2}^{2}\right) .
$$

It is useful to think of $\lambda$ 's as $2 \mathrm{D}$ vectors in a half strip $\Lambda=$ $\mathbb{R}^{+} \times\left\{\sigma^{2} \leq \epsilon^{-2} /(2 \pi)\right\}$, and the function $\bar{x}$ as an element in $\overline{\mathcal{X}}_{\epsilon}: \Lambda \rightarrow \mathbb{R}$, that defines an isocontours for each value of $\bar{x}$.

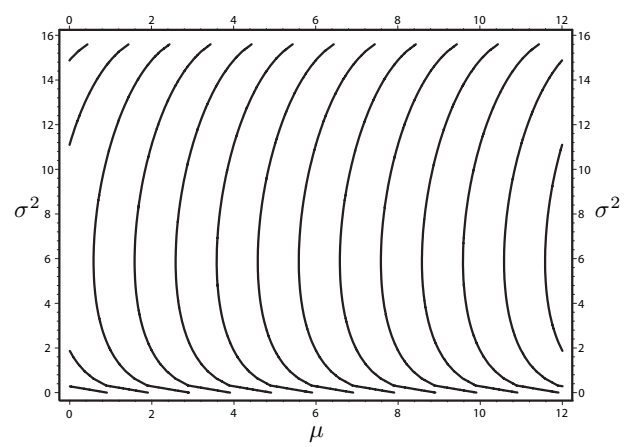

Fig. 1: Isocontour profile of $\bar{x}$ on $\sigma^{2}$ versus $\mu$.

For example, in Figure 1, the contour plot for $\epsilon=0.1$ is shown. The contour increases from left to right, and at $\sigma^{2}=0$, the value $\bar{x}$ reaches its limit of $\mu$. If we denote $G$ 's maximum path variance by $\sigma_{\max }^{2}$, then we must have $\sigma^{2} \leq \sigma_{\max }^{2}=$ $\epsilon_{\max }^{-2} /(2 \pi)$ so that for all $\boldsymbol{\lambda} \in \Lambda$, the value $\bar{x}$ exists. For now, we claim that $\sigma_{\max }^{2}$ is computed with the longest path algorithm on $G$ with edge metrics $\sigma_{i}^{2}$ and usual addition. Later, we show that finding $\sigma_{\max }^{2}$ is crucial to guarantee closure on $\Lambda$.

\section{Generalized DijKstra's Algorithm}

Having defined the channel model for each communication link, we now introduce the Generalized Dijkstra's Algorithm (GDA) below [2]. The algorithm can operate on any metric of choice as long as the metric obeys a set of algebraic properties. Specifically, in this paper we would like to show that the $\lambda$ and $\bar{x}$ metrics of the AWGN channel defined in the previous section is compatible with the GDA.

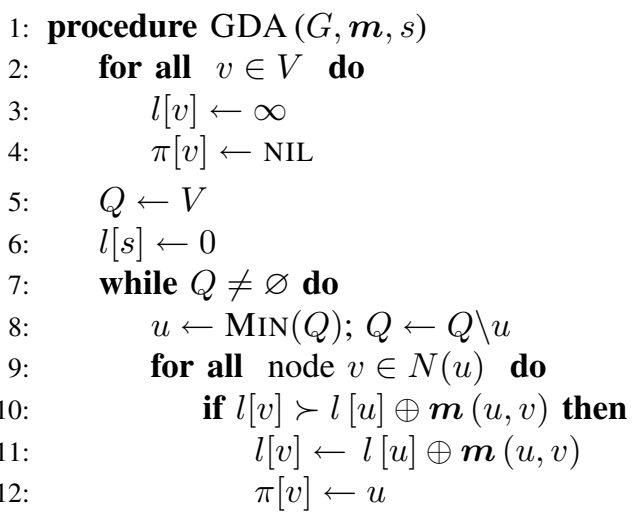

Upon examination, the GDA is practically identical to the Dijkstra's Algorithm (DA) except for the relaxation step, where the $\oplus$ and $\preceq$ operators act on a generalized metric space $\mathcal{M}$ (instead of the equivalent step in DA, where + and $\leq$ operators act on $\mathbb{R}$ ). We shall shortly define $\preceq$ for our channel.

On line $9, N(u)$ denotes the set of all nodes adjacent to $u$. The argument $\boldsymbol{m}$ is the lengths of the edges in $G$ each of which is an element in $\mathcal{M}$, and $\boldsymbol{m}(u, v)$ is the length of $\langle u, v\rangle$. Lines 10-12 perform the relaxation step of the GDA. This step depends on the definitions of $\mathcal{M}, \oplus$, and $\preceq$. If the GDA (in)correctly returns the path in $G$ with minimum length measured in $\mathcal{M}$, then $(\mathcal{M}, \oplus)$ and $\preceq$ are said to be (in)compatible with the GDA. The following is the required properties for compatibility:

Proposition 1: An algebra $\mathbf{A}=(\mathcal{M}, \oplus)$ and a total order $\preceq$ is compatible with the GDA if and only if it satisfies all the properties in the set denoted by $\mathbf{P}$ below:

P1 is a commutative monoid, that is, for $a, b, c \in \mathcal{M}$ :

- $\mathcal{M}$ is closed under $\oplus: a \oplus b \in \mathcal{M}$;

- $\oplus$ is associative : $a \oplus(b \oplus c)=(a \oplus b) \oplus c$;

- 0 is the identity : $a \oplus 0=0 \oplus a=a$;

- $\oplus$ is commutative : $a \oplus b=b \oplus a$.

P2 There exists $\infty \in \mathcal{M} \mid a \oplus \infty=\infty \oplus a=\infty$.

P3 $\preceq$ is a total order on $\mathcal{M}$, i.e., $\preceq$ is :

- reflexive: $a \preceq a$;

- anti-symmetric: if $a \preceq b$ and $b \preceq a$ then $a=b$;

- transitive: if $a \preceq b$ and $b \preceq c$ then $a \preceq c$;

- total: for every $a, b \in \mathcal{M}$ either $a \preceq b$ or $b \preceq a$.

P4 There exists the least element 0 that satisfies $0 \preceq a$. P5 $a \oplus c \prec b \oplus c$ if $a \prec b$ and $c \in \mathcal{M}-\{\infty\}$.

PROOF: Refer to [2] for a complete proof.

Next, we prove that the $\mathbf{B}$ algebra we defined for the constrained AWGN channel is compatible with the GDA. 
The proof requires us to define and use $\preceq$ to compare any $\lambda, \boldsymbol{\lambda}^{\prime} \in \Lambda$. Since $\Lambda \subset \mathbb{R}^{2+}$ and $\mathbb{R}^{2+}$ is not a totally ordered set, we must define $\preceq$ in terms of $\bar{x}(\boldsymbol{\lambda}, \epsilon)$ as follows:

$$
\begin{aligned}
\boldsymbol{\lambda} \preceq \boldsymbol{\lambda}^{\prime} \Leftrightarrow & \bar{x}(\boldsymbol{\lambda}, \epsilon)<\bar{x}\left(\boldsymbol{\lambda}^{\prime}, \epsilon\right) \text { or } \\
\bar{x}(\boldsymbol{\lambda}, \epsilon) & =\bar{x}\left(\boldsymbol{\lambda}^{\prime}, \epsilon\right) \text { and } \sigma^{2} \leq \sigma^{\prime 2}
\end{aligned}
$$

Graphically, this means that the $\bar{x}$ isocontours defined on $\Lambda$ determines whether $\boldsymbol{\lambda} \prec \boldsymbol{\lambda}^{\prime}$. If they lie on the same contour line, then the variances break the tie. We also define $\Lambda$ as follows:

$$
\Lambda=\left(\mathbb{R}^{+} \times\left[0, \sigma_{\max }^{2}\right] \cap\left\{\boldsymbol{\lambda}: \frac{\mu}{\sigma^{2}} \geq\left.\frac{\partial \mu}{\partial \sigma^{2}}\right|_{\sigma_{\max }^{2}}\right\}\right) \cup\{\infty\}
$$

$\Lambda$ is depicted by the shaded region in figure 2 . For a fixed value of $\sigma^{2}$, along any $\bar{x}$ contour, the slope $\mu^{\prime}=\partial \mu / \partial \sigma^{2}$ is equal for all $\mu$ and is maximized at $\sigma_{\max }^{2}$. If $\boldsymbol{\lambda}, \boldsymbol{\lambda}^{\prime}$ have slopes larger than $\mu^{\prime}$, their sum lies in a higher contour.

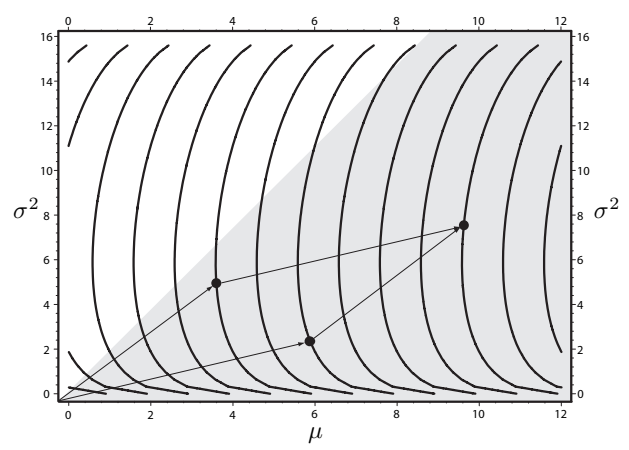

Fig. 2: The metric space $\Lambda$ (shaded).

Theorem 2: The algebras $\mathbf{B}=(\Lambda, \oplus)$ defined for the AWGN channel and its total order $\preceq$ satisfy all the properties in $\mathbf{P}$, and thus compatible with the GDA.

PROOF: P1 Except for closure, the other monoid properties are obvious because $\oplus$ is the standard vector addition. Because $\Lambda$ does not occupy the full non-negative octant, but rather bounded by $\sigma^{2} \leq \sigma_{\max }^{2}=\epsilon_{\max }^{-2} /(2 \pi)$, in general closure is not guaranteed. However, if we can compute:

$$
\sigma_{\max }^{2}=\max _{\pi \in \Pi}\left\{\sum_{i: v_{i} \in V_{\pi}} \sigma_{i}^{2}\right\}
$$

then closure is guaranteed. This value of $\sigma_{\max }^{2}$ can be obtained by running the DA once on $G$, costing $O\left(V^{2}\right)$. In case one (or both) of the operands is $\infty$, then as in q-SC and q-EC, by definition, the $\oplus$ sum is also $\infty \in \Lambda$.

P2 The proof is derived from closure on $\infty$.

P3 The proof follows from the definition of $\Lambda$ and $\preceq$.

P4 Both terms in equation (5) are minimized when they are zero, i.e., $\mu=0$ and either $\sigma^{2}=0$ or $\sigma^{2}=\epsilon^{-2} /(2 \pi)$. However, only $\boldsymbol{\lambda}=(0,0) \in \Lambda$, and is thus the 0 element.

P5 Obvious from figure 2 and the definition of $\Lambda$.

From the definition of $\boldsymbol{\lambda} \preceq \boldsymbol{\lambda}^{\prime}$, it follows that a path's $\bar{x}$ value is a non-decreasing function of its $\lambda$ values, which means that the path with minimum $\boldsymbol{\lambda}$ is also the path with minimum $\bar{x}$.

\section{Optimal Worst-Case QoS Routing}

The preceding assertion - that $\bar{x}$ is a non-decreasing function of $\boldsymbol{\lambda}$ - allows us to construct the optimal worst-case QoS routing algorithm. The path algebra for $\lambda$ 's is relatively simple. In the case of our constrained AWGN channel, since the edges are independent, the $\oplus$ operation is simply a vector addition. In contrast, the algebra for $\bar{x}$ depends on computing $\lambda$ first. First, we argue for the need of an optimal worst-case QoS routing algorithm that routes information through error correction nodes by proving our earlier claim:

Proposition 3: If $V_{\pi_{J}} \cap U=\varnothing$, then $\beta\left(\pi_{j}\right)$ is an nondecreasing function of $j$. The minimum $\beta\left(\pi_{j}\right)=0$ is only possible if the edge lengths $p_{j}=0$ for all $j=0 \ldots J$.

Proof: P5 with $0=a \prec b=p_{j}$, and $c=p_{\pi_{j-1}}$ gives us $\beta\left(\pi_{j-1}\right)=p_{\pi_{j-1}} \prec p_{\pi_{j-1}} \oplus p_{j}=\beta\left(\pi_{j}\right)$, proving that $\beta\left(\pi_{j}\right)$ is a non-decreasing function of $j$. The second part of the proof can be derived directly from $\mathbf{P 1}$.

The preceding proposition shows the practical benefit of including error correcting nodes in the network. If the path includes $u \in U$, then for some $j \in[0, J]$, we can have $\beta\left(\pi_{j}\right)=0$ even with $p_{j} \neq 0$ for all $j$. This feature could be added into most existing shortest-path based routing protocols.

Suppose these protocols compute the path based on $\bar{x}$. A path $\phi=\left\langle v_{1}, v_{2}\right\rangle$ is feasible iff $\omega\left(\left\langle s, v_{1}\right\rangle\right) \oplus \omega(\phi) \leq x_{\max }$ - the value of $\bar{x}$ up to $v_{1}$ added to the $\bar{x}$ of $\phi$ must be less than $x_{\max }$. Denote by $\Phi \subseteq \Pi$ the feasible paths in $\Pi$, and by $\Phi\left(v_{1}, v_{2}\right)$ the feasible paths between $v_{1}, v_{2}$.

Theorem 4: A path $\pi^{*}$ is the path with minimum $\omega(\pi)$ iff it solves the Shortest Path Problem (SPP) given by $G^{\prime}=\left(V^{\prime}, E^{\prime}\right)$, where $V^{\prime}=\{s\} \cup U$.

An edge connecting two nodes $v_{1}, v_{2} \in V^{\prime}$ is the shortest path in $\Phi\left(v_{1}, v_{2}\right)$, i.e.,

$$
E^{\prime}=\left\{\operatorname{argmin}_{\phi}\left\{\omega(\phi) \mid \phi \in \Phi\left(v_{1}, v_{2}\right)\right\} \mid v_{1}, v_{2} \in V^{\prime}\right\}
$$

PROOF: Suppose $\pi^{*}$ contains $n+1$ segments $\phi_{i}$ connecting the nodes in $V^{\prime \prime}=\left\{s, U_{\pi^{*}}, d\right\}$, where $U_{\pi^{*}}=U \cap V_{\pi^{*}}$. In segment notation, $\pi^{*}$ is denoted by $s \rightsquigarrow u_{1} \rightsquigarrow \cdots \rightsquigarrow u_{j} \rightsquigarrow d$, with $\left\{u_{i}\right\}=U_{\pi^{*}}$, and $0 \leq j \leq|U|$.

Then $\phi_{i}$ must be the shortest feasible paths between adjacent nodes in $V^{\prime \prime}$, and $U_{\pi^{*}}$ must be the set that minimizes $\sum \beta\left(\phi_{i}\right)$. Otherwise, a better path $\xi^{*}$ can be obtained by modifying $\phi_{i}$ or $U_{\pi^{*}}$, contradicting the claim that $\pi^{*}$ is optimal.

For the forward proof, note that $V^{\prime \prime}=\left\{s, d, U_{\pi^{*}}\right\} \subseteq V^{\prime}$. Further, since each $\phi_{i}$ is a shortest path between nodes in $V^{\prime}$, then it has a representation in $E^{\prime}$, i.e., $\phi_{i} \in E^{\prime}$. Therefore $\pi^{*}$ is the solution to the SPP given by $G^{\prime}=\left(V^{\prime}, E^{\prime}\right)$.

For the reverse proof, suppose $\xi^{*}$ is the SPP solution but is not the minimum $\bar{x}$ path $\pi^{*}$. From the forward proof, if $\pi^{*}$ minimizes $\bar{x}$, then it also solves the SPP, thus $\omega\left(\pi^{*}\right) \leq \omega\left(\xi^{*}\right)$.

However, if $\omega\left(\pi^{*}\right)<\omega\left(\xi^{*}\right)$, then $\xi^{*}$ is not the SPP solution - a contradiction. Thus, $\omega\left(\pi^{*}\right)=\omega\left(\xi^{*}\right)$, and if path lengths are unique, $\pi^{*}=\xi^{*}$. Hence, the SPP solution $\pi^{*}$ is also the minimum $\bar{x}$ path. 
Theorem 4 essentially proves the correctness of the optimal worst-case QoS routing algorithm listed below. Although in this paper $\bar{x}$ is linked to the worst-case value of $x$ for the constrained AWGN channel, other worst-case metrics can be used.

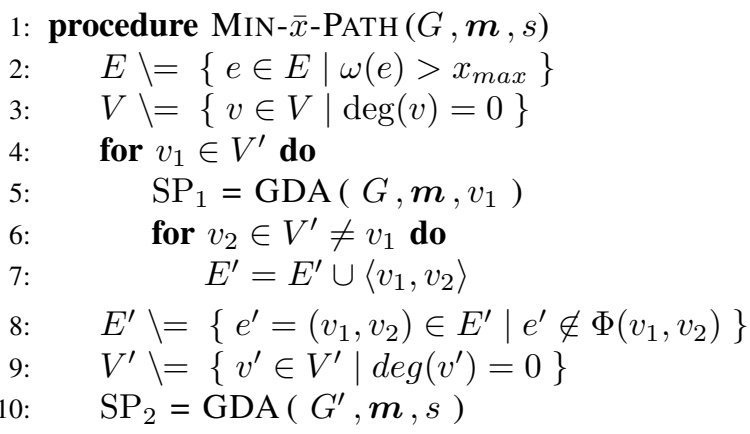

On lines 2 and 3, the algorithm prunes all infeasible edges. Then, on line 5, it runs the GDA on all $v_{1} \in V^{\prime}$, every time producing a shortest path tree $\mathrm{SP}_{1}$ rooted at $v_{1}$. On line 7, the edges connecting $v_{1}$ and $v_{2} \in V^{\prime}$ are added into $E^{\prime}$ based on $\mathrm{SP}_{1}$. This finishes the first stage and starts the second stage. On lines 8 and 9, the infeasible edges in $E^{\prime}$ are pruned, and any isolated nodes in $V^{\prime}$ are removed. On line $10, \pi^{*}$ is finally calculated using GDA.

Let us denote $\left|V^{\prime}\right|$ by $\alpha$. The first stage produces a complete graph with $\alpha$ nodes and $\alpha(\alpha-1)$ directed edges by executing the GDA $\alpha$ times on line 5, and thus has a time complexity of $O\left(\alpha V^{2}\right)$ (if the GDA is implemented using Fibonacci heap, then its complexity could reach $O(V \log V+E)$ [18]).

Lines 8 and 9 search linearly over them with $O\left(\alpha^{2}\right)$ time complexity. The GDA on line 10 has a time complexity $O\left(\alpha^{2}\right)$. Hence, overall time complexity is $O\left(\alpha V^{2}+\alpha^{2}\right)$, or conservatively, $O\left(V^{3}\right)$.

\section{CONClusion AND Discussion}

In this paper, we extended the optimal worst-case QoS routing algorithm and metric definition given in [1]. We proved that in addition to the $q$-ary symmetric and $q$-ary erasure channel model, the necessary and sufficient conditions defined in [2] for the Generalized Dijkstra's Algorithm can be used with a constrained non-negative-mean AWGN channel.

The generalization allowed the computation of the worstcase QoS metric value for a given edge weight density. We showed that this worst-case value can then also be used as the routing metric. This also allowed an exact analysis for the case where some network nodes have error correcting capabilities. The result is an optimal worst-case QoS routing algorithm that uses the Generalized Dijkstra's Algorithm as a subroutine with a polynomial time complexity of $O\left(V^{3}\right)$.

Future work includes supplementing the theoretical results with simulation and experimental verification. We believe there are still many important probability densities that are compatible with this routing framework. Specific implementation of this algorithm into existing and future protocols is also of interest. Finally, the parameter $\epsilon$ can be used to evaluate the worst-case performance of real life communication networks.

\section{REFERENCES}

[1] Soedarmadji, E., "Optimal Routing in the Worst-Case-Error Metric," Proc. of IEEE Int'l Conf. on Communications Systems 2006.

[2] Sobrinho, J.L., "Algebra and algorithms for QoS path computation and hop-by-hop routing in the Internet," IEEE/ACM Transaction on Networking., vol. 10, pp. 541-550, August 2002.

[3] Kurceren, B. et al., "A joint source-channel coding approach to network transport on digital video," Proc. of INFOCOM 2000, vol. 2, pp. 717-726, 2000.

[4] Michelson, A.M. et al., "Reliable ATM transmission of multimedia in wireless networks," Universal Personal Comm., 1998, vol. 2, pp. 10211027, 1998.

[5] Nikaein, N. et al., "MA-FEC: a QoS-based adaptive FEC for multicast communication in wireless networks," Proc. of IEEE ICC, 2000, vol. 2, pp. 954-958, 2000.

[6] Wang G.J. et al., "Channel-adaptive error protection for scalable video over channels with bit errors and packet erasures," Proc. of ISCAS 2002, vol. 2, 712-715, 2002.

[7] Barsocchi, P. et al., "Experimental Results with Forward Erasure Correction and Real Video Streaming in Hybrid Wireless Networks," 2nd International Symposium on Wireless Communication Systems, 2005, pp. 662-666, 2005.

[8] Huitema, C., "The case for packet level FEC," in Proceedings of IFIP PfHSN 1996.

[9] Nonnenmacher, J., et al., "Parity-based loss recovery for reliable multicast transmission," IEEE/ACM Transactions on Networking, vol. 6, no. 4, pp. 349-361, 1998.

[10] Lancerica, L., et al. "Evaluation of content-access QoS for various dissemination strategies in peer to peer networks," Proceeding of IEEE ICON 2003, pp. 337-342, 2003.

[11] Byers, J., et al., "Informed Content Delivery Across Adaptive Overlay Networks," Proceedings of ACM SIGCOMM, 2002.

[12] Kostic, D., et al., "Bullet: High Bandwidth Data Dissemination Using an Overlay Mesh," Proc. of ACM SOSP, 2003.

[13] Wang, M., et al., "A High-Throughput Overlay Multicast Infrastructure with Network Coding," Proceedings of IFIP IWQOS 2005 pp. 37-53.

[14] Du Q., et al., "Adaptive Low-Complexity Erasure-Correcting Code-Based Protocols for QoS-Driven Mobile Multicast Services," Proceeding of QShine 2005, pp. 29-29, 2005.

[15] Li Q. et al., "Providing adaptive QoS to layered video over wireless local area networks through real-time retry limit adaptation," IEEE Transactions on Multimedia, vol. 6, no. 2 pp. 278-290, 2004.

[16] Li J., et al., "A practical proactive hybrid FEC/ARQ technique for reliable multi-session multicast communication," Proceeding of IEEE ICECS 2003, vol. 2, pp. 519-522, 2003.

[17] Stanica, P., "Good Lower and Upper Bounds on Binomial Coefficients," Journal of Inequalities in Pure and Applied Mathematics., vol. 2, no. 3, article 30, 2001.

[18] Cormen, T.H.; Leiserson, C.E.; Rivest, R.L., Introduction to Algorithms, MIT Press, MA and McGraw-Hill, NY, 1990. 